\title{
New version of Eugene Shoemaker formula more suitable for calculating the impact energy of meteorites and methods for calculating the mass and volume of meteorites
}

\author{
Huadong D. Zhao ${ }^{1}$ Weigao Li $^{1}$ Haishan Dang ${ }^{2}$ Jundong Tian ${ }^{3}$
}

1. Luohe Medical College, Luohe 462002

2. Wuhan Botanical Garden, the Chinese Academy of Sciences, Wuhan 430074

3. College of Life Sciences, Zhengzhou University, Zhengzhou 450001

[Abstract] For an impact crater on land, scientists often need to estimate the impact energy according to the diameter of the impact crater. Since there is no unified formula to describe the complex physical process of impact, the results estimated by different scholars are quite different, which makes it difficult to judge which one is more consistent with the facts. For impact craters in the history of the earth, which have been unable to know the impact angle, there is an urgent need for a unified formula to obtain a more pertinent calculation result.After comparing and analyzing several formulas put forward by previous scientists, we think that Eugene Shoemaker's formula in 1990 is better. We simplify and improve it in order to make it adapt to the calculation of very large impact energy, so as to avoid fallacies. At the end of the paper, we also give a general method to estimate the mass and volume of meteorites after obtaining the impact energy. The improved formula and method proposed in this paper can be used for reference by scholars and science lovers, and may need further research to improve it in the future.

[Key words] meteorite impact; impact energy; new version of Eugene Shoemaker's formula; mass and volume of meteorite; estimation of Bermuda Impact Event

\section{Introduction}

Calculating the energy produced by a meteorite hitting the earth is a problem that scientists in related fields often encounter, and it is also a topic of interest to science lovers. However, it is a very complex problem. Up to now, there is no unified calculation formula in the world, and the parameters that need to be considered in each specific calculation are not exactly the same. Different scholars' estimates to the same impact crater could vary greatly, sometimes tenfold. So there is an urgent need for a unified formula to obtain a more pertinent calculation result.

One of the most common cases of this problem is that the diameter of the impact crater is known to solve the impact energy. We compared and analyzed the calculation formula of impact energy put forward by several previous scientists. We think that the 1990 Shoemaker's formula is the best one. In order to make the formula more convenient to calculate the huge impact energy, we have improved and converted it, and obtained a new version of the formula. By inputting the diameter length of the crater described in kilometers, we can use this formula to get the impact energy with million tons of TNT. We hope that this formula can be widely used by the scientific community in estimating large-scale impact energe.

\section{Our improved version of Eugene Shoemaker's formula}

There is no an exact unified formula for calculating the energy of meteorite hitting the earth in the academic circle, and the difference between the results obtained by different formulas may be

Correspondence and requests for materials should be addressed to Huadong D. Zhao (email: huadong_zhao@yeah) 
more than an order of magnitude. Corresponding author: Zhao Huadong Dian, E-mail:

huadong_zhao@yeah.

According to the energy formula for the large craters (4 $\mathrm{km}$ in diameter or higher) from Shoemaker, E. M. in $1983^{[1]}$ and $1990^{[2]}$, giving it a Gault, D.E. ${ }^{[3]}$ angle correction, using the average incidence angle ${ }^{[2]} 45^{\circ}$, ignoring the difference in the densities of the alluvial soils between the Bermuda impact site and the current Nevada nuclear test site, we finally got the simplified formula version as follows:

$$
E=6.286 D^{3.4}
$$

In formula (1), the unit of energy $E$ is megaton TNT energy equivalent, 1 megaton TNT energy equivalent $=4.184 \times 10^{15} \mathrm{Joule}$, and $D$ is the diameter of the impact crater represented by $\mathrm{km}$.

Next, we review several different formulas for estimating the impact energy since 1977, and then give the derivation of equation (1) :

\section{Several formulas and evaluations have been proposed in history 2.1 Formula given in 1977 by M. R. Dence}

In 1977, Dence, M. R. ${ }^{[4]}$ gave the formula for calculating the energy released by an impact event with a crater larger than $2.4 \mathrm{~km}$ in diameter:

$$
D_{R}=1.96 \times 10^{-5} E^{1 / 3.4}
$$

Where $D_{\mathrm{R}}$ is in $\mathrm{km}$ and $E$ is in joules. If the diameter $D_{R}$ of the impact crater is replaced by $\mathrm{D}$, and the unit of energy $E$ is converted into millions of tons of TNT energy equivalent, the following equation can be obtained:

$$
E=\left(\frac{D_{R}}{1.96 \times 10^{-5}}\right)^{3.4}=\frac{\left(\frac{1}{1.96 \times 10^{-5}}\right)^{3.4}}{4.184 \times 10^{15}} \cdot D^{3.4} \approx 2.425 D^{3.4}
$$

Thus, the Dence's simplified impact formula, in which $D$ expressed as $\mathrm{km}$ and $E$ expressed as million ton energy equivalent of TNT - is as follows:

$$
E=2.425 D^{3.4}
$$

As can be seen below, the energy calculated by this formula is relatively small.

\subsection{Formula given by E. M. Shoemaker in 1979}

In 1979, Shoemaker, E. M. ${ }^{[5]}$ gave the relationship (S-2) between the diameter of the impact crater and the energy released, where $D_{e} \geq 3 \mathrm{~km}$, the unit of $D_{e}$ is meter, and the unit of energy $W$ is kiloton TNT energy equivalent:

$$
D_{e}=74 \times 1.3 W^{1 / 3.4}
$$

Replace $D_{\mathrm{e}}$, unit of $\mathrm{m}$, with $D$, unit of $\mathrm{km}$; replace $W$, expressed in thousand tons of TNT energy equivalent, with $E$, expressed in million tons of TNT; take 1 million tons of TNT $=1000$ thousand tons of TNT into equation (S-2), the equation (S-3) can be obtained by equivalent deformation as follows:

That is:

$$
E=\frac{W}{1000}=\frac{\left(\frac{D_{e}}{74 \times 1.3}\right)^{3.4}}{1000}=\frac{\left(\frac{D \times 1000}{74 \times 1.3}\right)^{3.4}}{1000} \approx 2.87 D^{3.4}
$$

$$
E=2.87 D^{3.4}
$$


Formula (S-3) represents the early simple calculation formula of E. M. Shoemaker for the energy $E$ (million tons of TNT energy equivalent) released from a meteorite impact crater with a diameter of $D$ (unit is $\mathrm{km}, \mathrm{D} \geq 3 \mathrm{~km}$ ).

In 1994, Bergh, S. V. D. ${ }^{[6]}$ transformed formula (S-2) from E. M. Shoemaker 1979 into the following formula (where $D$ is in kilometers and $E$ is in ergs):

$$
\log D=\log E^{1 / 3.4}-6.77
$$

\subsection{Formula given by E. M. Shoemaker in 1983}

In 1983, Shoemaker, E. M. ${ }^{[5]}$ gave the relationship between the diameter of the meteorite impact crater and the energy released based on the nuclear test results as follows:

$$
D_{t}=C_{f} k_{n}\left(W \rho_{a} / \rho_{t}\right)^{1 / 3.4}
$$

$D_{t}$ is the diameter of the impact crater in $\mathrm{km} ; k_{\mathrm{n}}=0.074$; when $D \geq 4 \mathrm{~km}, c_{f}=1.3$; $W$ is the energy equivalent of kiloton of TNT; $\rho_{a}$ refers to the density of the alluvial soil of Nevada nuclear test site, $\rho_{t}$ refers to the soil density of meteorite impact site. If ignore the density difference between the crash site and the test site, then formula (S-4) becomes:

$$
D_{t}=0.074 \times 1.3 W^{1 / 3.4}
$$

Formula (S-5) is completely equivalent to formula (S-2), both them can be transformed into formula $(\mathrm{S}-3)$.

\section{The derivation process of E. Shoemaker-formula simplified version: $E=$ 6.286 $D^{3.4}$ in this research}

In 1990, Shoemaker, E. M. ${ }^{[2]}$ improved and generalized the equation (S-4) on the surface of solid planets in the entire solar system into the following equation:

$$
D=0.074 \cdot C_{f}\left(g_{e} / g\right)^{1 / 6} \cdot\left(W \rho_{a} / \rho_{t}\right)^{1 / 3.4} \cdot D_{i}
$$

$D$ is the diameter of the impact crater, in $\mathrm{km}$; when $D \geq 4 \mathrm{~km}, c_{f}=1.3 ; g_{e}$ is the gravitational acceleration of the earth, $g$ is the gravitational acceleration of the planet being hit; $W$ is the energy equivalent of kiloton of TNT; $\rho_{a}$ refers to the alluvial soil density of the Nevada nuclear test site, $\rho_{t}$ is the density of the impact site of the planet involved; $D_{i}$ is the impact angle correction factor added later by Shoemaker, he adopted Gault's formula (in 1973) ${ }^{[3]}: D_{i}=$ $(\sin i)^{2 / 3}$, so the above equation becomes:

$$
D=0.074 \times 1.3 \cdot\left(g_{e} / g\right)^{1 / 6} \cdot\left(W \rho_{a} / \rho_{t}\right)^{1 / 3.4} \cdot(\sin i)^{(2 / 3)}
$$

If the impact site is on the Earth, thus $g_{e}=g$; in order to facilitate the estimation, we deliberately neglect the density differences between the soil of impact site and that of the Nevada nuclear test site, so $\rho_{a}=\rho_{t}$; the average incidence angle ${ }^{62} i=45^{\circ}$, the formula above comes into being:

$$
D=0.074 \times 1.3 \cdot W^{1 / 3.4} \cdot\left(\sin 45^{\circ}\right)^{(2 / 3)}
$$

$D$ is the diameter of the impact crater, in kilometers; $W$ is the energy equivalent in kilotons of TNT. If replace $W$ with $E$ in energy equivalent of megatons TNT, the formula above change into the follow:

$$
E=\frac{W}{1000}=\frac{\left(\frac{D}{0.074 \times 1.3 \cdot\left(\sin 45^{\circ}\right)^{(2 / 3)}}\right)^{3.4}}{1000} \approx 6.286 D^{3.4}
$$

Then simple version of E. Shoemaker's formula to estimate the impact energy is:

$$
E=6.286 D^{3.4}
$$


In formula (1), $E$ is the energy equivalent of megaton TNT, 1 megaton TNT equivalent $=$ $4.184 \times 10^{15}$ Joule, and $D$ is the diameter of the impact crater represented by $\mathrm{km}$.

\section{Illustrate that the calculation results using Shoemaker's simple formula $E=$ 6.286 $D^{3.4}$ is moderate through Chicxulub crater}

We take the Chicxulub impact crater as an example to illustrate that Shoemaker's simplified formula $E=6.286 D^{3.4}$ can be used to calculate a moderate result:

Originally thought that the Chicxulub crater's diameter is $180 \mathrm{~km}^{[7,8,9]}$, however, the energy released by Chicxulub impact event aroused a controversy. In 1997, Pope, K. O. ${ }^{[10]}$ pointed out the energy related to Chicxulub crater was in $0.7 \sim 3.4 \times 10^{31} \mathrm{erg}$, namely $(2.05 \pm 1.35) \times 10^{31} \mathrm{erg}$, in this interval, the maximum value is about five times the minimum value, and the deviation ratio: $1.35 / 2.05$ of the marginal value from the median value reaches astonishing $66 \%$.

Next, we use the simplified formula (1) to calculate the energy, take $D=180 \mathrm{~km}$ and $1 \mathrm{Mt}$ of TNT energy equivalent $=4.184 \times 10^{22} \mathrm{erg}$ into it, then we get:

$$
E=6.286 D^{3.4}=6.286 \times 180^{3.4} \times 4.184 \times 10^{22} \approx 1.22 \times 10^{31} \mathrm{erg}
$$

It can be seen that when the diameter is $180 \mathrm{~km}$, the energy value $1.22 \times 10^{31}$ calculated by Shoemaker's simplified formula is a little smaller than the median value $2.05 \times 10^{31}$. The deviation from the median value is $(2.05-1.22) / 2.05 \approx 40 \%$, and the approximation to the median value is $60 \%$.

Later, there were different voices about the size of the Chicxulub crater, in 1993, Pope, K. O. et al ${ }^{[11]}$, suggested the Chicxulub crater had a diameter of $240 \mathrm{~km}$ (Sharpton, V. L. et al. $[12,13,14]$ think the Chicxulub crater diameter is $300 \mathrm{~km}$, this value is very big, so we don't adopt it here).

Then, if the intermediate value: $D=210 \mathrm{~km}$ between $180 \mathrm{~km}$ and $240 \mathrm{~km}$ is substituted into Shoemaker's simplified formula (1), the following result can be obtained:

$$
E=6.286 D^{3.4}=6.286 \times 210^{3.4} \times 4.184 \times 10^{22} \approx 2.07 \times 10^{31} \mathrm{erg}
$$

It can be seen that the energy value $2.07 \times 10^{31}$ calculated by Shoemaker's simplified formula is only slightly larger than the median value $2.05 \times 10^{31}$. The deviation degree from the median value is (2.07-2.05)/2.05 is approximately $1 \%$, and the approximation degree to the median value is $99 \%$, which is in good agreement.

So we say that in the field of estimating the energy released by large impact craters on earth, the results from Shoemaker's formula simplified version are very relevant.

\section{Estimating the size of the Bermuda Impact Event and the mass and volume of the Bermuda Meteorite------includes general methods}

Take the diameter of the Bermuda impact crater ${ }^{[15]} D=2000 \mathrm{~km}$ into formula (1), we get: $E=6.286 D^{3.4}=6.286 \times 2000^{3.4} \approx 1 \times 10^{12}$ megaton TNT energy equivalent

So the Bermuda impact energy is $E \approx 1 \times 10^{12}$ megaton of TNT energy equivalent, 1 megaton TNT energy equivalent $=4.184 \times 10^{15}$ Joule.

We adopt the general method: according to the law of conservation of energy, the energy released by the Bermuda impact is equal to the kinetic energy carried by the Bermuda meteorite, so there are:

$$
E=0.5 m v^{2}=6.286 \cdot D^{3.4} \times 4.184 \times 10^{15}
$$

Based on equation (S-6), the mass of the Bermuda meteorite can be got. in (S-6), $E$ is the energy represented by joule. Given that $D=2000 \mathrm{~km}$ and the impact velocity $v=15000 \mathrm{~m} / \mathrm{s}$ of the ordinary meteorite, we can get: 


$$
m=\frac{2 \times 6.286 \cdot 2000^{3.4}}{15000^{2}} \times 4.184 \times 10^{15} \approx 3.9 \times 10^{19} \mathrm{~kg}
$$

The mass of the Bermuda meteorite is known as $m \approx 3.9 \times 10^{19} \mathrm{~kg}$.

If the Bermuda meteorite is regarded as a sphere, the density $\rho=2970 \mathrm{~kg} / \mathrm{m}^{3}$ of the asteroid of 511 Davida $^{[16]}$, whose mass is close to that of Bermuda, is chosen as the density of Bermuda, and the ordinary impact velocity $v=15000 \mathrm{~m} / \mathrm{s}$ is brought into the equation (2) below, we get the diameter of Bermuda meteorite:

$$
d=2\left(\frac{3 E}{2 \pi \rho v^{2}}\right)^{1 / 3}=2\left(\frac{3 \times 6.286 \cdot 2000^{3.4} \times 4.184 \times 10^{15}}{2 \pi \times 2970 \times 15000^{2}}\right)^{1 / 3} \approx 293 \mathrm{~km}
$$

The diameter of the Bermuda meteorite is $d \approx 293 \mathrm{~km}$, which is about 23 times the diameter of the Chicxulub meteorite. In formula (2), $E$ is the impact energy expressed by Joule, and the other parameters are also based on the International Unit System.

The following is the derivation of formula (2) :

If a meteorite is regarded as a solid sphere with regular shape and uniform density, using $d$ as the diameter of the sphere, and the volume of the sphere is $V=4 \pi(d / 2)^{3} / 3$; because the mass of the sphere is equal to the product of density and volume, i.e. $m=\rho V$, then the mass of the sphere $m=$ $4 \pi \rho(d / 2)^{3} / 3$; since the enormous energy contained in the high-speed meteorite is contained in its enormous kinetic energy, according to the kinetic energy theorem $E=m v^{2} / 2$, it is concluded that $E=2 \pi \rho v^{2}(d / 2)^{3} / 3$, that is formula (2). The process is summarized as follows:

$$
\left\{\begin{array}{c}
V=\frac{4}{3} \pi\left(\frac{d}{2}\right)^{3} \\
m=\rho V \\
E=\frac{m v^{2}}{2}
\end{array}\right\} \rightarrow E=\frac{2}{3} \pi \rho v^{2}\left(\frac{d}{2}\right)^{3} \rightarrow d=2\left(\frac{3 E}{2 \pi \rho v^{2}}\right)^{1 / 3}
$$

\section{Discussion}

Our improved formula and the method of getting the mass volume of meteorites are all general formulas based on classical mechanics. Since the parameters cannot be exactly the same for each impact, it is difficult to accurately calculate the impact energy of a meteorite, but it is still of important reference significance to use our improved formula. Since the speed of meteorites is much lower than the speed of light, there is no need to consider the relativistic effect. Of course, we still hope that more scientists will pay attention to the energy problem of meteorite impact, so as to further improve the calculation formula and method more in line with the actual situation.

\section{References}

[1]. Shoemaker, M.E. Asteroid and comet bombardment of the earth. Annual Review of Earth and Planetary Sciences 11, 461-494(1983).

[2]. Shoemaker, E. M., Wolfe, R. F. \& Shoemaker, C. S. Asteroid and comet flux in the neighborhood of the earth.in Sharpton, V. L. \& Ward, P. D., eds., Global catastrophes in Earth history; An interdisciplinary conference on impacts, vocanism, and mass mortality: Geological Society of America Special Paper 247(1990).

[3]. Gault, D. E. Displaced mass, depth, diameter, and effects of oblique trajectories for impact craters formed in dense crystalline rocks. Moon 6, 32-44(1973). 
[4].Dence, M. R., Grieve, R. A. F. \& Robertson, P. B. "Terrestrial impact structures - Principal characteristics and energy considerations." Impact and Explosion Cratering: Planetary and Terrestrial Implications Impact and Explosion Cratering: Planetary and Terrestrial Implications, 247-275(1977).

[5].Shoemaker, E. M. et al. Earth-crossing asteroids: orbital classes, population, and fluctuation of population in late geologic time. Reports of Planetary Geology Program (1979).

[6].Bergh, S. V. D., Astronomical Catastrophes in Earth History. Publications of the Astronomical Society of the Pacific 106, 689-695(1994).

[7]. Hidebrand, A. R. Chicxulub crater : a possible Cretaceous/Tertiary boundary impact on the Yucatan Peninsula, Mexico. Geology 19, 867-871(1991).

[8]. Pilkington, M., Hildebrand, A. R. \& Ortiz-Aleman, C. Gravity and magnetic field modeling and structure of the Chicxulub crater, Mexico. Journal of Geophysical Research Planets 99, 13147-13162(1994).

[9].Hildebrand, A. R., Pilkington, M., Connors, M., Ortiz-Aleman, C. \& Chavez, R. E. Size and structure of the Chicxulub crater revealed by horizontal gravity gradients and cenotes. Nature 376, 415-417(1995).

[10]. Pope, K. O., Baines, K. H., Ocampo, A. C. \& Ivanov, B. A.. Energy, volatile production, and climatic effects of the chicxulub cretaceous/tertiary impact. Journal of Geophysical Research-space Physics 102, 21645-21664(1997).

[11]. Pope, K. O., Ocampo, A. C. \& Duller, C. E.Surficial geology of the Chicxulub impact crater, Yucatan, Mexico. Earth Moon \& Planets 63, 93-104 (1993).

[12]. Sharpton, V. L. et al. Chicxulub multiring impact basin: size and other characteristics derived from gravity analysis. Science 261, 1564-1567(1993).

[13]. Sharpton, V. L., et al. A Model of the Chicxulub Impact Basin Based on Evaluation of Geophysical Data, Well Logs, and Drill Core Samples." Geological Society of America Special Paper (1996).

[14]. Urrutiafucugauchi, J., Marin, L., \& Trejogarcia, A. Unam scientific drilling program of Chicxulub impact structure-evidence for a 300 kilometer crater diameter. Geophysical Research Letters 23, 1565-1568 (1996).

[15]. Zhao Huadong Dian,Dang Haishan,Shen Xiaocheng,et al. Newfound Huge Latent Impact Structure Suggests an Extraterrestrial Cause for the End-Triassic Extinction[EB/OL].

Beijing:Sciencepaper Online[2020-09-25].

http://www.paper.edu.cn/releasepaper/content/202009-73.

[16]. Baer, J., Chesley, S. R., \& Matson, R. D. Astrometric masses of 26 asteroids and observations on asteroid porosity. Astronomical Journal 141(5), 143(2011). 